\title{
El comercio colonial español de la Carrera de Indias: historiografía y método en el análisis de una estrategia de redes/
}

\section{The Spanish Colonial Trade of the Carrera de Indias:} Historiography and Methodology of a Social Network Strategy

\author{
Ana Crespo Solana \\ ORCID iD: https://orcid.org/0000-0002-5807-7751 \\ Instituto de Historia, CSIC
}

Este ensayo, más que un estado de la cuestión exhaustivo, trata de exponer en líneas generales cuál ha sido la evolución de los estudios de redes mercantiles en el ámbito del comercio entre España y América durante los siglos XVI al XVIII. Se trata de apuntar aquellos marcos teóricos más sobresalientes partiendo de los estudios sobre familias de mercaderes y agentes, y resaltando los logros metodológicos y las posibilidades futuras de esta línea de investigación.

Palabras clave: Redes mercantiles; Análisis de Redes Sociales (SNA); Historia Global; Comercio Atlántico; Historia Conectada; Historiografía.

This essay, more than an exhaustive state of affairs, address how has been the evolution of the studies of merchant networks in the trade between Spain and America from the $16^{\text {th }}$ to $18^{\text {th }}$ centuries. The aim is to point out the most outstanding theoretical frameworks based on studies of families of merchant and agents by highlighting the methodological achievements and future of this line of research.

KeYwords: Merchant Networks; Social Networks Analysis (SNA); Global History; Atlantic Trade; Historiography; Entangled History; Historiography.

Copyright: (C) 2018 CSIC. Este es un artículo de acceso abierto distribuido bajo los términos de la licencia de uso y distribución Creative Commons Reconocimiento 4.0 Internacional (CC BY 4.0). 


\section{Redes e imperio: aproximación teórica en el caso del comercio atlántico español}

Una revisión que se precie sobre la situación actual de los estudios americanistas aludiría a conceptos como «redes complejas»o «arquetipo de mercader» para describir lo que en palabras de Enriqueta Vila Vilar fue «una epopeya nunca bien contada» de la colonización y el comercio con América, idea secundada por tantos otros especialistas de la historia social y económica de los intercambios atlánticos. En este marco los estudios de redes son una innovación aunque se presentan dispersos entre el relativamente amplio panorama de estudios sobre familias, agentes, grupos mercantiles, comunidades o colonias de extranjeros. ${ }^{1}$ Es esta una historiografía necesaria que aglutina prototipos humanos en el mapa de los agentes e identidades en movimiento que relacionaron las dos orillas. Hoy sabemos que la historia del comercio entre España y América, conocido como la Carrera de Indias, se basó en el despliegue de una estrategia de redes constituidas por una compleja estructura espacial y de agentes que sobredimensionaba las propias fronteras de imperios, reinos, encuadramientos político-administrativos y repúblicas. Las redes estaban constituidas por agentes, muchos de los cuales se dedicaban casi exclusivamente al comercio, aunque no solo, siendo objeto de pioneros trabajos que priorizaban el estudio de mercaderes sobre mercaderías y el movimiento del tráfico, como ya incluso algunos especialistas del comercio de Indias reivindicaban desde la década de $1980 .^{2}$ El análisis del comportamiento de estas redes, así como los agentes que las constituían, ha sido enfocado desde distintas perspectivas teóricas y metodológicas, aunque no por ello carentes de un hilo conductor, diacrónico, exhaustivo y comparado, examinado a lo largo de una versátil infinidad de trabajos de investigación. En un principio, y a veces aun hoy, se describe a los agentes encuadrados en el concepto de burguesía mercantil, un término que recalcaba las capacidades de expertos en la invención y manejo de las

1 Vila Vilar, 2012b, 183. Sobre este conjunto de temas relacionado con la funcionalidad de las redes y comunidades ya tuve oportunidad de realizar un estado de la cuestión en: Crespo Solana, 2011 y 2015. La historiografía sobre redes y agentes en el mundo hispano-americano es muy extensa y me remitiré a algunos de estos trabajos a medida que extraigo conclusiones generales; de ellos quizás los más actualizados sean el de Iglesias Rodríguez y García Bernal (2016) y el de Iglesias Rodríguez, García Bernal y Díaz Blanco (2018). Algunos de estos trabajos han sido publicados en la revista Anuario de Estudios Americanos, cuyos contenidos más relevantes publicados a partir del año 1974 cito a lo largo de estas páginas.

2 García Fuentes, 1982. 
prácticas económicas, dedicadas a profesiones relacionadas con la mar y que residían, por lo general, en las ciudades. ${ }^{3}$

El término burguesía parece haber quedado algo obsoleto, al menos en lo que respecta a las complejas redes en torno a la Carrera de Indias; y no sin razón, cuando el concepto es demasiado heterogéneo para describir la interrelación de los grupos sociales. Ello es especialmente interesante teniendo en cuenta que el marco histórico es una sociedad de Antiguo Régimen y colonial, donde paradójicamente coexistieron comportamientos corporativos y en red. Tal antagonismo se ha visto recreado en los estudios sobre grupos mercantiles, familias, colonias de extranjeros y redes sociales entre América y Europa, e incluso en la propia prosopografía histórica sobre financieros y negociantes, con mayor o menor intensidad. Las interacciones derivadas de los intercambios sociales y económicos no solo afectaron a España, los virreinatos y otros territorios ultramarinos o de las Antillas, sino que dieron lugar a importantes mecanismos inclusivos y de competencia con el resto de las naciones marítimas de la época, generando una historia geográficamente integrada, un mapa global de ciudades portuarias y regiones productivas, en definitiva, unos puntos nodales (nodal points) de redes transnacionales.

La teoría de redes en el mundo global ha sido explicada ampliamente por los teóricos de la historia económica, y en el marco historiográfico de la Carrera de Indias apunta a un universo complejo, casi articulado en torno a espacios informales de producción, comercio e intercambio y no menos de reproducción social, algo que tampoco era exclusivo de las redes de la Monarquía Hispánica aunque bien se puede decir que estas eran un modelo sin precedentes. ${ }^{4}$ Estas redes, auto-organizadas en su definición antropológica y socio-económica, ${ }^{5}$ integrada por agentes de diversas características socio-profesionales y socio-políticas, configuraron una red marítima

3 Sobre burguesía mercantil hay infinidad de trabajos que toman una directriz importante con la edición de una recopilación de ponencias titulada: La burguesía mercantil gaditana (1650-1868), con prólogo de José Antonio Calderón Quijano (Cádiz, 1976). Destacamos también: Socolow (1978) y Guimerá Ravina (1987). Una aproximación teórica y metodológica sobre el concepto de burguesía en Bustos Rodríguez, 2010b, y Aranda Pérez, 2003.

4 Molina e Irigoyen, 2009.

5 El concepto que mejor las define es self-organized networks que he descrito en anteriores trabajos míos, como: «núcleos complejos y flexibles que forman redes evolutivas integradas en el marco histórico correspondiente a los siglos de la Edad Moderna por comerciantes, productores, comunidades y funcionarios gubernamentales», o «redes comerciales flexibles de proyección internacional y basadas en relaciones de parentesco o negocios». Crespo Solana, 2010a, 25. Ver también Schulte Beerbühl, 2010 . 
que promovió la propagación de mecanismos para solucionar la falta de información, problema obvio en la evolución de una economía espacial, en mercados sobre productos, rutas y confianza para el conocimiento de socios e informantes. ${ }^{6}$ En realidad, el comercio colonial con América y la configuración de diversos universos o «sistemas» en torno al Atlántico constituyó una heterogénea espacialidad que difícilmente ha sido estudiada como una unidad histórica de análisis. ${ }^{7}$ La colonización de La Española en 1493 abrió un proceso de interacción social, entendida esta como sinónimo de expansión, que daría lugar a la emergencia de nuevas formas de comportamiento humano en respuesta a nuevos condicionamientos y por la necesidad de intercambio de recursos y medios de subsistencia.

La historiografía sobre las relaciones entre España y América, influida por la escuela francesa de los Annales y por las teorías macroeconómicas (lo cual se aprecia en las clásicas obras Séville et l'Atlantique de Chaunu y Cádiz y el Atlántico de García-Baquero),${ }^{8}$ ha tenido a sus protagonistas sociales como uno de los temas claves para comprender este complejo marco histórico; ha integrado estudios socio-institucionales; fueron consecuencia de una tradición de debates sobre los orígenes del Capitalismo; y entroncan con la línea de investigación sobre el mundo atlántico y la Global History. Además, los conocimientos que tenemos sobre redes de agentes en el comercio colonial hispano prácticamente caminan a hombros de gigantes al constituir las investigaciones actuales una natural evolución de los muchos trabajos existentes sobre familias de mercaderes, grupos (muchos de ellos extranjeros), elites y dinastías asentados en los centros comerciales y marítimos de España y América, conectados con las fuentes de riqueza, producción y comercio, así como con los centros de poder. Aunque los estudios de redes en el imperio español son muy heterogéneos, ofrecen esa imagen de imperio negociado que apuntaban Daniels y Kennedy, ${ }^{9}$ y que ha derivado en una importante masa bibliográfica sobre Monarquía Hispánica y élites de poder en torno a la articulación del mundo hispano-americano. ${ }^{10}$

6 Subrahmanyam, 1996. Curtin, 1984. El concepto nodal points ha sido utilizado en el caso del análisis de las redes mercantiles neerlandesas en el mundo atlántico (Oostindie y Roitman, 2014) y también en el análisis de las ciudades portuarias (Polónia y Rivera Medina, 2016).

7 Vila Vilar, 2012a, 27. Games, 2006.

8 García-Baquero, 1976. Chaunu, 1955-1960.

9 Daniels y Kennedy, 2002, 1-15.

10 La literatura existente sobre esta perspectiva de redes, instituciones y poder político es muy abundante tanto para América como para la parte española, incluso sin haberse usado el concepto de red, en algunos casos. Véase, por ejemplo: Vila Vilar y Kuethe, 1999; Recio Morales, 2012, 37-55; Ibarra y Valle Pavón, 2007, 16 y ss. 
Tanto el papel de las redes como la importancia del espacio en el cual se articulaban aquellas inciden en la fuerte persistencia de la cooperación entre agentes, pero sin dejar de lado la consideración del espacio y la percepción de las rutas con objeto de visualizar conexiones entre actores históricos y ciudades portuarias. Si algo caracterizó a las redes es que estas fueron dispersas y se distribuyeron en un espacio que se rigió por las normas intrínsecas de la colonialidad (entendiéndose como el conocimiento emanado entre los centros de poder y las relaciones intersubjetivas) y la interdependencia económica. Por ello destaca la necesidad de enfocar la interacción entre agentes sociales y geografía, analizando el peso específico de las distintas ciudades en los mapas, sobre todo en relación a los esquemas administrativos, económicos e institucionales de los imperios. Esta perspectiva bien podría ampliarse al estudio de cómo las diferencias geográficas, raciales, étnicas, religiosas o culturales impactan en la forma de entender las construcciones propias de la sociedad humanas tales como lo público, las instituciones, o los diversos asociacionismos o mutualismos humanos.

Las oportunidades del comercio marítimo produjeron la organización de viajes de comercio agrupados en convoyes y navíos sueltos desde varios puertos del Golfo de Cádiz y Sevilla, zona que se erigió como el centro neurálgico del impropiamente denominado monopolio. ${ }^{11}$ A mediados del siglo XVI se consolidó un sistema reglado de flotas y galeones, formalizado por orden de la Corona tras diversos debates acerca de su conveniencia y seguridad, y coincidiendo con la configuración política de las Indias occidentales y con la eclosión del importante mercado en torno a la plata americana a partir de la década de 1570. Estos hechos originaron el desarrollo de lo que historiográficamente se denomina Atlántico español, descrito de forma sistemática y objeto de amplios debates en los que no voy a profundizar ahora pero que sin duda apuntan a la existencia evolutiva de un circuito, región o espacio interrelacionado que era único en comparación con otras áreas geo-políticas y que no solo constituía un fenómeno económico sino también cultural. Aunque algunos trabajos no son realmente estudios de redes, una buena parte de los estudios de las últimas décadas se han basado en la aceptación de que las redes construyeron la sociedad mercantil que dio lugar a este sistema atlántico, el cual «va más allá de lo concreto geográfico para referirse a una red de relaciones predominantemente, aunque no

11 Oliva Melgar (2004) realiza una excelente descripción de las razones del «monopolio que nunca existió». 
exclusivamente, económicas». ${ }^{12}$ Sin duda, y aunque apenas se ha enfocado desde esta teoría, el Atlántico ibérico (especialmente durante la etapa de la Unión Ibérica con Portugal) constituyó un sistema económico espacial al presentar importantes rasgos de acuerdo a las teorías iniciadas por Thunen, Fujita y Krugmann, y que sirven para explicar importantes enigmas de las interacciones del comercio internacional, su evolución, y que guarda una importante relación con el Spatial Turn de la Historia Global. ${ }^{13}$ La aplicación de este contexto metodológico, especialmente con el uso de las nuevas tecnologías (o Sistemas de Información Geográfica), pone de relieve la capacidad del historiador para la extracción de una importante minería de datos con el fin de analizar los movimientos del intercambio con una exhaustiva ordenación, o la idea de la Historia como ciencia aplicada, pues: «Historians are quite good at determining a context for the interpretation of texts». ${ }^{14}$

El sistema espacial de las flotas y galeones así como el inmenso territorio administrativo vinculado permanecería con pocos cambios hasta mediados del siglo XVIII cuando las reformas borbónicas introdujeron novedades tanto en las formas de navegación como en las instituciones que, junto al estado continuo de guerra en el mar, produjeron importantes reorganizaciones en el mapa político y económico y, por ende, en la configuración de las redes. Por ejemplo, la evolución geoestratégica de las zonas vinculadas se vio afectada en varias coyunturas, especialmente entre las décadas de 1630 y 1680, cuando otras naciones europeas lograron establecerse en áreas marginales del imperio español, así como en otras zonas de América. Las redes fortalecieron sus lazos de relación con outsiders en un marco de comercio ilegal, ajeno a las exigencias político-legales y de piratería y contrabando. La aplicación de la teoría general de sistemas a la comprensión de este mundo complejo ha derivado en la apertura de interesantes revisiones basadas en la perspectiva del análisis de redes, aplicadas estas tanto a la comprehensión del espacio como a los comportamientos de los agentes.

Grosso modo, puede decirse que el sistema de flotas, galeones y navíos de registro, y su evolución durante tres centurias, condicionaron la espacialidad de las rutas, las formas de integración regional geográfica (desde el

12 Davis, 1973. Pietschmann, 1999. Bustos Rodríguez, 2005, 20. Emmer, 2008. 1999.

13 Pieper y Schmidt, 2005, 17-19. Sobre la economía espacial: Fujita, Krugman y Venables,

14 Wachowicz y Owens, 2012, 55. 
punto de vista de su espacialidad) y el comportamiento de las redes económicas, así como las acciones de los agentes mercantiles en relación a estas. Esta espacialidad estaba condicionada por tres aspectos fundamentales. En primer lugar, la zona marítima que estaba reglada meteorológicamente por los Trade Winds facilitó los viajes a través de una ruta que vinculó islas y territorios desde las Canarias y las Azores con las pequeñas Antillas primero y con el espacio del Caribe y Golfo de México, después. La zona afectada por los vientos alisios se convirtió en la vía principal de integración en los océanos Atlántico y Pacífico desde que este fenómeno meteorológico fue advertido por los navegantes portugueses en el siglo XV y más tarde por Andrés de Urdaneta en 1565 para el Pacífico. ${ }^{15}$ La reglamentación de las flotas de Indias se articuló en torno a las regiones vinculadas a estas zonas desde las que se tenía acceso a los dos virreinatos de México y Perú. En segundo lugar, el comportamiento de las redes en este espacio era causa, y consecuencia a su vez, del desarrollo de las instituciones, especialmente la Casa de la Contratación y los consulados de comercio en Sevilla y México, principalmente, que reglaron y también contravinieron los marcos legales según eventos y coyunturas. No se puede olvidar que las instituciones son el reflejo de las redes sociales, siendo ambas las dos caras de una misma moneda al ser la institución no otra cosa que la materialización física y política de la red.

Los estudios sobre redes sociales en la edad moderna muestran cómo los agentes sociales interaccionaban entre ellos creando, y no al revés, nuevos marcos institucionales, jurídicos y políticos en un contexto de reciprocidad social. Las redes se hacen visibles en la documentación histórica, aunque no siempre con la misma intensidad. Pasan etapas y experimentan cambios de comportamiento según los contextos. Las redes en torno a las instituciones llegaron a convertirse en lo visible mientras que paralelamente se producían redes invisibles integradas por agentes que influían desde espacios marginales y que podían incluso cambiar los acontecimientos políticos o económicos. Es casi un modelo a seguir por el resto de los lobbies mercantiles el comportamiento que desde el inicio del comercio con las Indias esgrimieron las elites sevillanas para gestionar e incluso manipular las funciones de Consulado de Cargadores desde que se aprobara la real provisión de julio de 1556, y que poco a poco permitiría que el consulado, por delegación de la Casa de la Contratación, asumiera funciones tendentes

15 Aunque Chaunu no utilizó el término trade winds, describe esta compleja estructura geográfica que condicionaría la Carrera de Indias. Ver Crespo Solana, 2014a. 
al control y regulación del tráfico marítimo ${ }^{16}$ Los consulados de México y Lima no fueron diferentes, dándose importancia a los análisis de sus componentes, al igual que en el caso metropolitano, su procedencia y condición social, sexo y procedencia geográfica o naturaleza. La omnipresencia de los mismos componentes familiares de estas redes llegó a ser transatlántica y transpacífica, entendiéndose así la proliferación de comportamientos similares en zonas tan aisladas. Tales procederes afectaron también a las políticas virreinales al generarse grupos de presión a cambio de financiaciones para los representantes del rey en el poder colonial, siendo este un esquema de comportamiento metropolitano que se repetía entre aristocracia y mercaderes también en el ámbito colonial. ${ }^{17}$ Las instituciones del comercio producían cambios en la logística comercial organizada por los propios mercaderes y con impacto en localizaciones centrales para la evolución propia del comercio como eran las ferias americanas. ${ }^{18}$ En el marco del comercio de Indias, la omnipresente Casa de la Contratación de Sevilla se encargaba, en teoría, de controlar la navegación y el comercio pero, a diferencia de lo que se ha dicho en relación a que articulaba un monopolio como pasaba con la Casa da India portuguesa, liberó el comercio a la inversión y a la iniciativa privada hacia $1505 .{ }^{19}$ Es interesante señalar cómo la ingente burocracia generada por el sistema comercial, orientada sobre todo a salvaguardar los derechos reales y la aplicación de gravámenes, ha llevado a la idea de que había una especie de monopolio cuando en realidad lo que la Corona pretendía era controlar los flujos de capital y los intereses privados en sus acciones y transacciones. ${ }^{20}$

En tercer lugar, la propia naturaleza de las actividades económicas en torno a la Carrera de Indias fue consecuencia de un proceso desarrollado por sus propios protagonistas sociales e institucionales, los agentes involucrados en los negocios. Esto abriría un profundo abanico socio-profesional, caracterizado también por la emergencia de nuevos oficios y sectores económicos, incluyéndose las finanzas, la construcción naval, los ejercicios de intermediación y otras tantas actividades portuarias. Por ello se usa la palabra agente y no mercader, aunque desde luego el comercio era, en dicho

16 García Fuentes, 1982, 25. Más tarde se reproducirían comportamientos semejantes en el Consulado de Cádiz: Ruiz Rivera, 1988 y 1990; Bustos Rodríguez, 2017.

17 Yuste, 1991 y 2007. Latasa, 2003.

18 Álvarez Nogal, 2011. Esta estrategia se veía también en los consulados de México, Lima, Sevilla y luego Cádiz: Vila Vilar, 2012, 192 y ss.; Ibarra y Valle Pavón, 2007; Studnicki-Gizbert, 2000.

19 Bernal, 2003.

20 Brendecke, 2012, 171. 
amplio universo de posibilidades, la forma de vida de los agentes que residían en las ciudades de la Carrera de Indias. En este escenario, las redes, desde su perspectiva analítica actual, constituyeron un sistema total en donde participaron individuos y grupos sociales diversos, de índole y procedencia dispar, rompiendo siempre la tradicional teoría de la edad moderna como una sociedad de compartimentos estancos y de estamentos.

En definitiva, red e imperio son dos cuestiones relacionadas en la historiografía cuando esta se ha vuelto Global History. ${ }^{21}$ Sin duda el imperio influyó en la conformación social en las ciudades marítimas y áreas portuarias más importantes, como sucedió en algunos casos como Veracruz, Cádiz, Sevilla, La Habana o Cartagena de Indias. Estos centros también actuaron como espacios donde nuevos grupos como los judíos-portugueses, negros y mulatos libres se insertaron en la sociedad americana través de las redes del comercio internacional, desempeñándose como mercaderes, pequeños comerciantes, navegantes, marineros, grumetes, arrieros, carreteros, etc. Según los grupos humanos que se asentaban en cada ciudad predominaba un tipo de comercio, unos determinados productos y unas preferencias por zonas de comercio y rutas. Estos grupos se convirtieron en intermediarios entre agentes y navegantes de distinta procedencia y ruta. Las redes influyeron en cómo un determinado puerto se erige de forma hegemónica, como pasó en el caso de Veracruz y Acapulco integrando lo que se ha llamado bioceanidad. ${ }^{22}$

\section{Espacialidad de las flotas y galeones y la formación de las redes}

El Atlántico hispano era un espacio heterogéneo producto de muchas localizaciones y lugares que formaba una Entangled History, que supera la idea nacionalista historiográfica y que emana de una visión antropológica y de análisis socio-cultural para comprender los procesos de globalización y el crecimiento de las interdependencias. Este aumento de los intercambios fue desproporcionado y no homogéneo. Para los historiadores y arqueólogos marítimos, el mejor espejo de esta globalización fue el barco, un espacio constreñido donde se reproducían mecanismos de redes de cooperación

21 Gruzinski, 2004. Aram y Yun, 2014.

22 Sobre el término bioceanidad: Domínguez-Domínguez, 2018. Las interacciones de las redes en estas ciudades portuarias claves para el comercio se han analizado en trabajos como: Alcántara, 2016; Angulo y Aragón, 2016; Valle Pavón, 2012; Caula, 2013; Gascón, 2000. 
y que representaba una especie de espejo en miniatura de la globalización. El barco reflejaba la idea de un imperio global que presentaba características espaciales que implicaban, a su vez, la ausencia relativa de fronteras permanentes y exclusivas. Sin duda, la articulación de este espacio imperial y marítimo mostraba una naturaleza paralela a la propia configuración fragmentada y compuesta de la Monarquía Hispánica, con sus diferentes reinos y territorios, con su estatus periférico que con el tiempo se fue consolidando y fragmentando desde el punto de vista de la riqueza y el poder a través de un proceso de elitización de gobernantes y autoridades locales, y con una condición policéntrica donde, sin embargo, no había relaciones independientes entre las entidades periféricas. ${ }^{23}$ Esta categorización de imperio y monarquía condicionó a los distintos grupos sociales que tanto en América como en Europa se vieron en la circunstancia de tener que reformularse a sí mismos ante la complejidad del nuevo espacio político y cultural que se formaba. ${ }^{24} \mathrm{~J}$. H. Parry describía cómo este imperio español poseía una maritime life-line que constituyó uno de los sistemas comerciales oceánicos que más han impactado en los movimientos globales. ${ }^{25}$ Todos los territorios de la Monarquía Hispánica no participaban por igual en el comercio con América. Desde la perspectiva legal, y desde las primeras décadas de la expansión ultramarina, la dinastía de los Austrias fijó una serie de condicionamientos para permitir esta participación que en parte evolucionó dependiendo de ciertas coyunturas y que afectó a las formas de participación de las redes, autóctonas de cada territorio y reino, extranjeras y criollas. La sistematización de las flotas de Nueva España y los galeones de Tierra Firme, así como el resto de las armadas destinadas a la protección tanto de la ruta comercial como de los territorios del imperio, estuvo focalizada a integrar espacialmente los territorios más importantes para la Corona en lo que a recursos naturales y financieros se refiere. ${ }^{26}$ Pero eso que se llamó monopolio era en realidad un organigrama institucional que pretendía dos cosas principalmente: imponer un centro de dominación geográfica al poder y la riqueza, por un lado, y por otro, reglamentar, ordenar y fiscalizar el comercio privado que se hacía desde España a las colonias y en el cual participaron varias escalas del espectro social de la época, especialmente

23 Cardim et al., 2012. Kaps y Komlosy, 2013. Kaps, 2016.

24 Yun Casalilla, 2009, 11-35.

25 Parry, 1990, 117-122.

26 La historiografía sobre el tema es muy extensa, aquí se ha utilizado: Mira Caballos, 1998, $17-21$. 
nobles y aristócratas, así como muchos comerciantes y gentes de negocios autóctonos y de origen foráneo. Una buena parte de estos participantes en el comercio colonial español fueron una mano de obra transmigrante y transeúnte, que se establecieron en colonias mercantiles o naciones a lo largo de muchas ciudades portuarias a donde llegaron a bordo de los buques mercantes. En un trabajo anterior describí el concepto de naciones como micro sociedades «en evolución con el objeto de integrarse y adaptarse a nuevos y dinámicos social environments». ${ }^{27}$

Muchos de estos comerciantes extranjeros se integraron en las elites locales y lograron, a corto plazo, influir en las decisiones y en las regulaciones comerciales. ${ }^{28}$ A pesar de cierta xenofobia es verdad que los mercaderes extranjeros alimentaban las flotas, ante la falta habitual de mercaderías suficientes que correspondiese a la demanda y hasta de barcos, y eran los primeros interesados en mantener las flotas regulares, como la historiografía ha reconocido. Antonio M. Bernal aludía a la verdad tras la permanencia de estos mercaderes en el sistema hispano a pesar de las guerras y las leyes de la Corona: «a los comerciantes internacionales les seguía siendo más favorable todavía acceder al comercio colonial español por dicha vía (la de la intermediación) que intentar el modo directo con las colonias». ${ }^{29}$ Así, el sistema se caracterizó por una socialización importante del comercio a través de las actividades de sus recursos humanos y su capital social.

En realidad el marco teórico-metodológico de los estudios de redes ya fue iniciado por especialistas de la historia atlántica y global como Philip Curtin y Frederic Mauro, que abrieron la perspectiva de la idea de diásporas mercantiles, redes de comerciantes y manufactureros asentados en una amplia red de lazos étnicos, lingüísticos, culturales y religiosos, que han influido notablemente sobre todo en la historiografía posterior sobre las comunidades y sociedades comerciantes. ${ }^{30}$ En este contexto es necesario definir qué es una red, discutida en varias ocasiones desde la perspectiva del análisis histórico. Aparte de la aplicación de las teorías sociológicas y la conceptualización de las redes auto-organizadas (Self-Organizing Network) que se ha descrito anteriormente, el concepto de red aparece claramente perfilado en recientes trabajos de investigación. La definición intrínseca a los brillantes comentarios de Eric Van Young a una recopilación de ensayos

27 Crespo Solana, 2015, 58.

28 Gamero Rojas, 2016.

29 Bernal, 2005, 189.

30 Curtin, 1984. Mauro, 1990. Trivellato, 2009. Caracausi y Jegle, 2014. 
sobre redes hispano-americanas implica una unificación teórica como agentes interesados en negocios comunes, que a veces se ven forzados a operar de forma mutualista y cooperativa en un ambiente de exclusión social. ${ }^{31}$ Relacionado con la conceptualización de red está la diversidad metodológica aun bajo el prisma del debate interdisciplinar y de foco geográfico, como se demuestra en la obra coordinada por Herrero Sánchez y Kaps, que también profundiza en una definición que coloca a las redes como los protagonistas absolutos del intercambio de productos y por tanto de las desigualdades en la economía y en la división del trabajo, dada la naturaleza del capitalismo mercantil y la necesidad de relacionar modos económicos previos con las exigencias del comercio a larga distancia. Pero ello describe a las redes, una vez más, como conectores de imperios. ${ }^{32}$

Empero, la definición de análisis de redes (SNA, siglas de Social Networks Analysis) y en concreto, de red, parte de definiciones simplificadoras usadas tradicionalmente en la sociología. Como concepto, la red social surge como la representación y medida de las relaciones y flujos entre personas, grupos, organizaciones, o entidades en general. Estas entidades se representan mediante nodos (agentes, ciudades) y sus relaciones mediante aristas (relaciones, rutas).$^{33}$ Estas estructuras constituyen puntos de conexión entre mundos, nodal points en el concepto adoptado en la revisión historiográfica para el caso de las redes de neerlandeses en el comercio global. ${ }^{34}$ Las conexiones de estas redes tienen importantes consecuencias para los individuos y para el sistema en su totalidad. En el mundo social esta aplicación deriva de la comprensión del mundo como un «mundo pequeño», según la teoría matemática de Watts y Strogatz, que fue adoptada por la escuela sociológica de Harvard. ${ }^{35}$ La teoría del mundo pequeño y la proliferación de lazos entre redes locales que tienen impacto en el «mundo grande» está influyendo en la visión del papel de las redes mercantiles en el mundo colonial español por diversas razones: en primer lugar, por la importancia dada a la difusión de la información que marcó el inicio del enfoque de redes. Segundo, esta teoría sociológica permite un modelado

31 Van Young, 2011, 290-293.

32 Herrero Sánchez y Kaps (2017, 1-37) hacen un brillante estado de la cuestión donde citan a los principales teóricos de las causas y consecuencias de la globalización económica de los siglos XVI al XVIII. Para el caso de estudios sobre redes en la España interior: Pérez Sarrión, 2012.

33 Krebs, Valdis, «Social Network Analysis: An Introducion», www.orgnet.com/sna.html [Consultado: 01/10/2012].

34 Oostindie y Roitman, 2014

35 Strength-of-weak-ties, teoría de la fortaleza de los lazos débiles. Granovetter, 1983. 
más sofisticado y realista de las estructuras de redes al explicar la conducta social humana, introduciendo además técnicas de visualización y análisis de datos. El análisis económico parte de la hipótesis de que toda interacción está embebida en las relaciones sociales, ideas ya desarrolladas por la clásica escuela de los Annales que han producido una importante teoría para el rol social en el análisis de los grupos de agentes en el comercio atlántico hispano-americano. Por último, la aplicación de las Social Networks Analysis se está produciendo por la influencia de otras disciplinas (como la Sociología, Psicología Social, la Antropología, las Matemáticas, o las Ciencias de la Información Geográfica, SIGs), ${ }^{36}$ o por la consideración de la Historia y la Arqueología como disciplinas aplicadas en el campo de las Ciencias Sociales que implican la consideración de las redes como constituyentes de datos relacionales que pueden ser definidos como un conjunto de entidades (individuos, grupos, organizaciones u otras), con relaciones o interacciones entre ellos.

En los estudios históricos que enfocan la sociedad, las instituciones y los comportamientos económicos, se han hecho análisis donde se recogen también interesantes estados del arte sobre esta aplicación. Es sobre todo en la perspectiva económica en donde la investigación histórica ha adoptado las herramientas de análisis de redes, no solo para el estudio de las conductas mercantiles sino de la logística de los espacios y rutas, por lo que es fundamental para conocer cómo se integran y evolucionan los mercados. Una parte de estos estudios describe estas actuaciones, pudiéndose extraer las siguientes características generales de la red: El impacto de la formación de redes en el espacio articulado por la Carrera de Indias condicionó las vías de migración y comercio, la labor misionera, el traspaso de tecnología y el intercambio biológico, pero también las conquistas militares. Algunas partes de la red sirvieron como puntos de interacción cotidiana ya sea en centros cosmopolitas o en ciudades portuarias. Las redes se esparcieron en espacios terrestres y marítimos, casi siempre constituidos por una serie de conexiones más cortas con docenas de ciudades portuarias ejerciendo de puntos de transmisión y transbordo. Las redes tienes diferentes escalas tanto en extensión como en densidad. Hacen que las prácticas se difundan con rapidez, reducen la diversidad cultural, promueven la especialización de la

36 SIG es la sigla de Sistema de Información Geográfica: un conjunto de herramientas que integra y relaciona diversos componentes (usuarios, hardware, software, procesos) y que permiten la organización, almacenamiento, manipulación, análisis y modelización de datos. Se está aplicando en los estudios de Historia Moderna. Crespo Solana, 2013. 
producción y la división del trabajo y fomentan la diversidad económica. Ello hace que las comunidades se especialicen (en torno a actividades de comercio, finanzas o producción agrícola), permiten explotar los recursos de forma más eficiente pero también más agresiva con el medio ambiente. En el caso de los mercaderes de la Carrera de Indias se identifican procederes orientados específicamente a controlar y gestionar los bienes importados y a veces incluso parece haber una lógica separación de funciones entre autóctonos y extranjeros. Compraban a través de agentes, vendían a altos créditos a cargadores españoles o consignaban ilegalmente los bienes a unos agentes que les servían de testaferros para que viajasen en las flotas a vender los productos en las ferias. La propia configuración de los mercados en Europa alentó las inversiones lejanas, de esta forma descrita ya por Braudel, y confirmada posteriormente a través de un elevado número de casos de estudio. ${ }^{37}$

Las redes hacen que las sociedades sean más ricas, pero también más jerárquicas, es decir, fomentan la desigualdad social y el recelo étnico y religioso. Como indicaban Bertrand, Guzzi-Heeb y Lemercier, estos trabajos «muestran una gran inventividad [sic] en materia de construcción de las fuentes, de las representaciones gráficas, de los cálculos de indicadores y/o de las interpretaciones para proponer una aproximación propiamente histórica de sus objetos de estudio». ${ }^{38}$ Los actores sociales, ya sean individuos, organizaciones o naciones, configuran su vida cotidiana a través de la consulta, la información y el intercambio de recursos, la sugerencia, el apoyo y la desaprobación de otros. Las interacciones en la red influyen en las creencias y actitudes, así como en el comportamiento, la acción y los resultados. Las redes sociales pueden ser distintas entre sí debido a cuestiones diversas como su estructura (tamaño, densidad, tipos de relaciones), contenido (fluidez, canales para la transferencia de comportamientos) y función. Las redes pueden entrar en conflicto con otras, siendo las interacciones sociales positivas o negativas, útiles o dañinas, integrando a los individuos en una comunidad y, igual de poderosamente, aplicar estrictas normas de aislamiento sobre el comportamiento.

Las estrecheces en interacciones sociales se han visto, por ejemplo, en grupos étnicos, donde pueden darse oportunidades de empleos restringidas. Mas no es necesariamente mejor con respecto a los lazos sociales. Como señaló Durkheim, demasiada supervisión (regulación) o apoyo (integración) puede ser sofocante y represiva. Además, los lazos «fuertes» no son

37 Stein y Stein, 2002, 28. Álvarez Nogal, 2011, 60. Alonso García, 2016.

38 Bertrand, Guzzi-Heeb y Lemercier, 2011. 
necesariamente óptimos porque los lazos «débiles» a menudo actúan como un puente hacia la información y los recursos diferentes ${ }^{39}$ y los agujeros en las estructuras de red proporcionan oportunidades que pueden ser explotadas..$^{40}$ Las redes a través de todos los niveles son estructuras y procesos dinámicos, no estáticos. La capacidad de formar y mantener vínculos sociales puede ser tan importante como su estado en un momento dado. Una perspectiva de red permite e incluso requiere enfoques multimétodo. La investigación cuantitativa es poderosa, documentando los efectos de las redes sociales, pero solo cuando está acompañada por la investigación cualitativa que describe por qué operan y miran de la manera que lo hacen. De ahí es cuando pasamos de la teoría a la reflexión práctica tomando las herramientas de redes para el análisis de este mundo. Es cierto que los estudios de historia moderna han aplicado más la teoría que los modelos de redes. ${ }^{41} \mathrm{De}$ hecho Bertrand, Guzzi-Heeb y Lemercier indicaban que aun constituían una especie de moda sin diálogo científico añadido. Para evitar este problema es necesario un previo conocimiento básico de los componentes de la red: nodos, átomo social, actores (sean individuales o institucionales); conexiones entre actores o links (ties); la existencia de subgrupos cuando el foco está en un grupo determinado de actores dentro de la red, con el objeto de plantear cuestiones de investigación relacionadas con el acopio de datos históricos. Las condiciones para establecer un modelo de análisis histórico de red se han de basar siempre en la cantidad de información recogida que, por ser una gran cantidad, debe ser procesada en bases de datos generadas siempre en torno a una topología (función de la red) cuya estructura en gran parte obedece a ese modelo y nos ofrece interesantes reflexiones sobre las implicaciones prácticas del análisis de redes. ${ }^{42}$ La sociología histórica ve la red social como la esencia misma de la estructura social, de los estados y naciones, a niveles micro y macro. Una cuestión previa fundamental es aceptar la teoría del mundo como una red o sistema complejo que implica que las relaciones de intercambio se producen más allá de las propias relaciones didácticas directas e incluye el intercambio indirecto y los distintos mecanismos de reciprocidad. Esto se contrapone a las primeras aproximaciones teóricas basadas en conjuntos institucionales o colectividades.

39 Granovetter, 1983.

40 Hace referencia al concepto Structural holes, también usado en los estudios históricos recientes. Para el caso de los mercaderes de Liverpool: Haggerty, 2017.

41 Bertrand, Guzzi-Heeb y Lemercier, 2011.

42 Sobre Wattz y Strogatz ver Marcelín-Jiménez, 2016. 


\section{De mercader a familia, dinastía y red}

La articulación de redes en el mapa espacio-temporal del Atlántico ibérico es clave para entender el despliegue de agentes productores de mecanismos de integración de áreas comerciales-coloniales. El análisis de estas redes, aparte de su encuadramiento teórico debe hacerse con una visión interdisciplinar con la aplicación de las SNA. Sin duda, el comercio de Indias es un campo de experimentación atractivo para esta metodología pero los estudios sobre redes mercantiles en la actualidad son el producto generado por una larga trayectoria científica de estudios sobre familias, comunidades y grupos sociales, sin dejar de lado la rica prosopografía histórica de los hombres y las mujeres de la Carrera de las Indias. ${ }^{43}$ Esta bibliografía es extensa y seguidora de una trayectoria concreta, los estudios de redes en la época moderna y colonial son parciales dada la complejidad que alcanzan las redes sociales y la cantidad de información que se debe recoger, aunque esta literatura es una extraordinaria generadora de Big Data para su empleo en bases de datos y SIGs. Las líneas más tratadas por estos trabajos de investigación pueden sintetizarse como se detalla a continuación.

En primer lugar, la naturaleza de la Monarquía compuesta ha enfatizado el estudio de las elites desde la perspectiva de la historia política y social, introduciéndose aquí también el concepto y método de red, pero viéndose a veces como algo ajeno a los grupos económicos, como si comercio y corte e instituciones fueran dos cosas ajenas. Elites y redes mercantiles tienen cosas en común, como se ha demostrado: movilidad geográfica, importancia de lazos familiares y clientelares, emergencia de nuevas formas de relación entre áreas lejanas gracias a la proliferación de vías de información y su influencia en el desarrollo de mercados, los viajes de comercio y los agentes (y servidores o representantes viajeros), etc. ${ }^{44}$ Algunas redes mercantiles, como la de los genoveses, fueron proveedoras de servicios a la aristocracia y a la Corona por lo que lograron establecerse en los accesos económicos e institucionales claves del imperio. ${ }^{45} \mathrm{En}$ definitiva, las redes se han visto analizadas también desde una perspectiva teórica relacionada con la idea de imperio, aunque algunos autores no siempre van en la misma dirección, con diversas teorías sobre el papel

43 Quizás un ejemplo de gran categoría sean los trabajos de Enriqueta Vila sobre la familia Corzo y Mañara, por ser un clásico de la biografía histórica del mercader de Indias (Vila Vilar, 1991).

44 Gamero Rojas, 2016. Gervais, Lemarchand y Margairaz, 2014. Yun Casalilla, 2009, 23.

45 Herrero Sánchez et al., 2011. 
de las redes sociales en la mediación y transmisión de información; ${ }^{46} \mathrm{o}$ los que enfatizan el rol socio-económico-espacial de las mismas sin dejar de lado sus descripciones político-culturales y sus identidades (más recurrente en los estudios sobre comunidades mercantiles y compañías de comercio); ${ }^{47} \mathrm{o}$ en las relaciones entre alta nobleza y mercaderes con el fin de extraer ventajas políticas. ${ }^{48}$

Los comportamientos de las redes del imperio se trasladaron muy pronto al ámbito americano, repitiendo o reproduciendo esquemas a través de las redes sociales tejidas en torno a los matrimonios o gracias al papel de la mujer como transmisora de valores. ${ }^{49}$ En la relación intrínseca existente entre aristocracia, política y comercio, algunos estudios se han enfocado en cómo mercaderes, funcionarios gubernamentales y autoridades coloniales desarrollaron amplias redes de colaboración, la mayoría invisibles, para comerciar en la clandestinidad, creándose una verdadera dinámica de luchas y rencillas para obtener el máximo poder posible, tanto en la faceta administrativa como en la comercial. En este tipo de análisis se aprecian dinámicas, muy documentadas, de rivalidades para colocar a hombres de confianza en aquellos oficios públicos más rentables, pero también para que estos contactos ahora bien situados facilitasen a otros miembros de la red una mayor seguridad para embarcar mercancías sin registro, contrabandear o llevar a cabo actividades paralelas a las legalizadas. Relacionando comercio y política se aprecia cómo interactúan los actores sociales en la construcción del proceso histórico, ello reflejado en modelos de estudio donde las biografías y los acontecimientos protagonizados por agentes explican la estructura histórica como algo no estático sino dinámico, especialmente en casos de conflictos bélicos. ${ }^{50}$ Los hombres de negocios también participaron en la dinámica cortesana, estatal, ilustrada y reformista de la Monarquía, siendo el análisis de redes la forma adecuada para calibrar hasta qué punto fueron germen de cambio. Estas propuestas conceptuales y de método se basan en datos sobre movilidad social y geográfica, estrategias patrimoniales y de matrimonio, educación, modelos culturales, círculos de sociabilidad y afinidades políticas. Se estudian también las influencias de estos grupos en sus sociedades de origen, incluyendo modos de vida y prácticas de patronazgo,

46 Gruzinski, 2004.

47 García Fernández, 2005. Lamikiz, 2010. Crespo Solana, 2010b. Iglesias Rodríguez y García Bernal, 2016. Crailsheim, 2016.

48 Guerrero Elecalde, 2012. Latasa, 2003.

49 Cachero Vinuesa, 2013.

50 Bernardo Ares, 2009, 11-17. 
comprobándose en muchos casos la relación entre paisanaje, los negocios y la lealtad a la Corona de la que obtenían beneficios, un mapa familiar que extiende la red más allá del paisaje. En este campo se ha llevado a cabo el análisis relaciones en el estudio de las relaciones de parentesco y la jerarquía de las elites así como los mecanismos de reproducción social. ${ }^{51}$

Otro capítulo de este sistema de redes lo protagonizaron las comunidades extranjeras asentadas en ciudades portuarias hispanas, sobre todo aquellas que estaban estrechamente conectadas a la Carrera de Indias. En un principio, la historiografía de las comunidades mercantiles se ha basado en los estudios socio-institucionales, principalmente consulados, y en el análisis de las relaciones de los mercaderes con los poderes políticos, aunque los estudios microeconómicos de familias y compañías particulares de comercio, vienen ofreciendo la mayor parte de los conocimientos que se tienen sobre las colonias foráneas. La presencia de extranjeros en el comercio colonial se remonta al inicio mismo de la navegación con América y era un hecho paralelamente proporcional a la dependencia que se tenía en España y sus colonias de las manufacturas del norte de Europa. En el caso andaluz, por ejemplo, Bustos Rodríguez subrayó que a falta de estudios sobre el comercio interior apenas hay indicios de la funcionalidad de los agentes mercantiles a la hora de la extracción de productos regionales, básicamente agrícolas, que constituían el tercio de frutos, o los textiles autóctonos, superados siempre por las manufacturas extranjeras. ${ }^{52}$ Los agentes mercantiles de origen extranjero tenían un indiscutible papel en este flujo de intercambios. Eran almaceneros de productos y capital, lo cual los relacionaba directamente con el consumo y las finanzas. También eran consignatarios de barcos y actuaban como pequeños banqueros locales y en el caso de Cádiz, como vendedores ilegales de plata. Su papel económico no se podía separar de las características de su particular integración socio-cultural y de la idea que tenían sobre ellos mismos como «nación». ${ }^{53}$ Desde los comienzos de la regulación de la navegación con América se intentó, sin resultado, controlar y fiscalizar su participación en un largo proceso legislador que con altibajos sufrió además las contingencias de las necesidades reales del intercambio. Pero como buen sistema basado en el fraude y el contrabando

51 Imízcoz Beunza, 2009. Guerrero Elecalde, 2012. Molina e Irigoyen, 2009.

52 Bustos Rodríguez, 2010a. Una recopilación amplia de trabajos sobre el papel de las comunidades de diversos orígenes en la economía hispana y americana: Villar García y Pezzi Cristóbal, 2003.

53 Crespo Solana, 2014b. Fernández Chaves y Gamero Rojas, 2009. 
las leyes contra extranjeros no llegaron a ser efectivas en la gran mayoría de los casos, especialmente porque gracias a las propias relaciones intrínsecas de simbiosis dentro de las redes había mecanismos suficientes para evadir el control. Oliva Melgar realiza una adecuada síntesis de estas leyes en relación a la forma en que funcionaba el comercio de Indias, y los muchos mecanismos han sido también ampliamente descritos en la bibliografía del tema ${ }^{54}$ Pero en este proceso legislativo imperó de forma general alguna que otra permisión, como la pragmática de 1623 con la que se permitía a los «extranjeros destos Reynos (como sean católicos y amigos de nuestra Corona)», puedan ejercer sus trabajos en España siempre que fuese «viviendo veinte leguas de la tierra adentro de los puertos». ${ }^{55}$

Este tipo de leyes, repetido durante los Austrias y también en el siglo XVIII, demuestra las preocupaciones de las elites gobernantes sobre los extranjeros y las inquietudes de los grupos sociales autóctonos: los extranjeros y la confesión católica, y la residencia de estos en zonas cercanas al mar y por ende con ánimos de participar (algo teóricamente prohibido para ellos, salvo exenciones especialmente fiscales) en el comercio y los viajes a América. Ello nunca se cumplió y a lo largo de tres siglos se produjeron muchas querellas entre la Casa de la Contratación y los mercaderes extranjeros, pero no muchas menos que las que se producían entre los ciudadanos locales que pretendían controlar la navegación y el comercio y saltarse las exigencias hacia la Corona. Los estudios han dado una singular importancia a las familias derivándose más tarde a las comunidades y grupos. Desde los trabajos de Enrique Otte y Enriqueta Vila Vilar, se ha producido una rica historiografía, culminada con trabajos sobre comunidades mercantiles, algunos de los cuales son ejemplos de historia geográficamente integrada donde se analiza desde lo local a las conexiones con el mundo euroamericano. Una gran cantidad de estos agentes trabajaron en círculos en torno a unos determinados agentes claves que eran siempre negociantes (financieros y mercaderes al por mayor) o empleados en las instituciones, que consiguen alcanzar un estatus social mediante los negocios comerciales y el oportunismo social: religión, buena familia, nivel cultural, padrinazgo y protección social. Este comportamiento fue repetitivo en la mayor parte de las comunidades mercantiles establecidas en

54 Oliva Melgar, 2004, 74-76. García-Mauriño, 1999, 40-47.

55 Novísima Recopilación de las Leyes de España mandada formar por el Señor Don Carlos IV, 4 vols., México, Galván Librero, Portal de Agustinos-París, Rosa, Librero, 1831, tomo, II, libro VI, titulo XI, ley I, 167. 
distintas áreas marítimo-portuarias. Además de constituir una importante contribución teórica, estos estudios han generado una enorme cantidad de Big Data para aplicar herramientas de análisis de redes para el estudio sociológico y económico de la integración de estos agentes, asentamiento en la ciudad e integración en el mapa social de la Carrera de Indias gracias a sus relaciones y sus dispositivos institucionales. Se han estudiado las conexiones inherentes a la red, la microhistoria de relaciones privadas y semi-privadas. La reconstrucción de las redes comerciales formadas por mercaderes extranjeros es posible, en gran medida, por el análisis de las relaciones que estos mercaderes despliegan a través de sus propios procesos de naturalización. Además, durante sus procesos de naturalización hacen uso de testigos pertenecientes a un amplio abanico socio-profesional y de distinto origen geográfico. Sería demasiado extenso anotar qué tipo de análisis se ha hecho en estos trabajos, pero sí se pueden subrayar cuestiones generales como el ennoblecimiento de grandes comerciantes (una secuencia comportamental europea, por cierto), aunque en la Monarquía Hispánica, sobre todo en los siglos XVII y XVIII, la alta nobleza castellana se vio desplazada de los espacios de decisión a favor del ascenso de otras familias, esencialmente de foráneos (franceses, italianos, genoveses, irlandeses, flamencos), quienes se convirtieron en indiscutibles proveedores de dinero a la Hacienda Real. ${ }^{56}$

Durante el siglo XVIII destacaron nacionales de distintos territorios de la Monarquía, como la minoría aragonesa fiel a la causa de Felipe de Borbón, u otros grupos provenientes de la periferia peninsular (los «norteños»): asturianos, montañeses de Santander, vasco-navarros o riojanos, grupos que también fueron a América o fueron activos mercaderes desde Sevilla y Cádiz. ${ }^{57}$ Estos grupos se caracterizan por un patrón social con unas formas de actuación que presentan similitudes (árbol genealógico, identidades, familias, acceso a las instituciones, etc.). La naturaleza dinástica y de corte de las monarquías de los Austrias y de los Borbones contribuyó al ennoblecimiento y enriquecimiento de una elite de comerciantes y banqueros y, más adelante, incluso de comerciantes que no procedían de la nobleza originariamente. Ello se daba, por supuesto - por la propia naturaleza del patronazgo, la dependencia, el servicio - en la corte, pero también porque llegó un momento en que la Hacienda Real debía mucho dinero a estos individuos. Por ello, muchos derivan desde asentistas (negociantes que tienen

56 Sanz Ayán, 2015. Álvarez Nogal, 1998.

57 García Fuentes, 2003. Ruiz Rivera, 1993. 
una contratación pública con el Estado o la Corona, y que esperan una consignación, generalmente a cambio del suministro de algún servicio) a factores (que gestionan ellos mismos todo el negocio). Alcanzan posiciones sociales pero también levantan recelos, como los genoveses en la corte de Felipe III. Esta circunstancia, como se ha visto sobre todo en el caso de las colonias de extranjeros, produce que emerjan «redes invisibles» y mecanismos de aumento de grupo, Group augmentation (o técnicas de aumento del grupo con miembros potencialmente influyentes en lo económico, lo cultural, lo político), con la idea de tener contactos fuera de la red que sean estratégicamente aptos para hacerles mantener su influencia político-institucional. ${ }^{58}$ Ello también hace que se formen partidos, grupos de poder o lobbies económicos y financieros que llegan a detentar rentas, como sucedió con el caso de los juros en el siglo XVII.

Relacionado con estas actividades socio-económicas, un capítulo importante y novedoso en el marco de los estudios de redes y agentes consiste en hacer referencia a las teorías sobre la cooperación y el análisis del comportamiento mercantil. Ello aun se ha analizado de forma minoritaria y enfocada desde distintas perspectivas. ${ }^{59}$ Pero lo singular de esta acepción es que puede llevarse al terreno del estudio de los intercambios para comprender cómo las pequeñas alteraciones de comportamiento socio-económico en el seno de familias de mercaderes locales pueden o no determinar cambios en sistemas no lineales y producir un efecto mariposa en la multiplicación y en las formas de comportamiento de las redes mercantiles en espacios vinculados, aunque dichos espacios sean lejanos geográficamente. Todas las colonias de mercaderes tenían como denominador común la presencia de agentes viajeros que se desplazaban y navegaban con sus mercancías, algo que explica la transitoriedad de larga duración de muchos de los componentes de las colonias foráneas. Estos mercaderes expandían sus propios negocios pero a la vez se integraban en un universo social construido por otros comerciantes. ${ }^{60}$ En un primer momento se ha ahondado en la función de mercaderes y comunidades como conectores entre producción y consumo, especialmente por su relación con la llegada de la plata americana a Europa. ${ }^{61}$ Algunos casos de

58 «edes invisibles» es un concepto explicado en Picazo Muntaner, 2011, y Crespo Solana, 2015, 51. El término Group Augmentation en Crespo Solana, 2017, 85.

59 Kaps, 2016.

60 Crespo Solana, 2014b.

61 Bustos Rodríguez, 2008. 
estudios sobre comunidades de mercaderes extranjeros se han analizado y representado los mecanismos comportamentales en bases de datos y redes, ofreciéndose descripciones específicas con datos cuantitativos reales, representaciones cartográficas y diversos marcos teórico-sociológicos que han evolucionado desde el estudio de la extensión de las redes a niveles sociales y espaciales desde la perspectiva de la sucursalidad y el padrinazgo, la teoría económica del trust o las recientes aportaciones desde la idea de la Network reciprocity (reciprocidad dentro de la red, que no es lo mismo que trust), y de la Group Augmentation. ${ }^{62}$ Sin duda, la clave para comprender las estrategias evolutivas de las redes mercantiles en el espacio y el tiempo es la cooperación en sus amplias acepciones teóricas. Los estudios sobre la cooperación han atraído mucho interés reciente y en el caso de las sociedades humanas llama la atención el desconocimiento que se tiene sobre qué es exactamente y porqué se produce, incluyéndose aquí parámetros de estudios sobre el altruismo recíproco, el mutualismo, la simbiosis o la necesidad del trading o intercambio. Todas ellas tienen en común que exploran interacciones recíprocas y dan lugar a la posibilidad de visiones comparativas entre casos de estudio que destacan cooperación y funcionalidad. Esto se ve claro en el caso de las colonias de mercaderes extranjeros. Mientras que la funcionalidad de algunos mercaderes era la de ser consignatarios y almaceneros de mercancías y capitales, y ahí era donde radicaba su sucursalidad, ${ }^{63}$ otros crearon sociedades mixtas, inversiones de diverso tipo incluso en sectores de servicios. Unos y otros constituyeron formas de cooperación mercantil en el seno de la sociedad. ${ }^{64}$ También los mercaderes genoveses y franceses presentaban semejantes pautas de funcionalidad y sus agentes prácticamente tenían comportamientos sociales oportunistas que lograron adoptar prácticas nuevas o imitar procedimientos y operaciones que a ellos les parecían apropiados. Muchos de los mercaderes transmigrantes y transeúntes no eran, en realidad, grandes hombres de negocios o banqueros y a veces procedían de la periferia social de la ciudad de origen de cada uno. Eran gentes que adaptaban sus acciones a la oportunidad del momento, razón además de sus pautas transmigratorias y continuos viajes. Se solían aglutinar en torno a un individuo que por sus influencias o su posición social era favorable a los negocios y que se constituía en el núcleo principal de una determinada red. En el

62 Crespo Solana, 2017.

63 Se ha comprobado, por ejemplo, para el caso flamenco y holandés: Crespo Solana, 2010b.

64 García Fernández, 2005, 138. 
marco de las altas finanzas y la Carrera de Indias, los negociantes buscan monopolios basados en productos con demanda, consolidando el monopolio asegurándose las vías, sobre todo marítimas, negociando asientos o contratos estatales, negociando con metales preciosos y promocionando el oligopsonio dentro de su red o comunidad además de relacionarse con las instituciones de Indias.

\section{Conclusión}

A modo de last words, una conclusión sobre el estado actual de los estudios de redes no se hace imperativa en el marco de este ensayo pues abarcaría demasiado para el espacio que rige aquí. La bibliografía citada en este ensayo no es más que un ejemplo de la singular tradición en la que ya se han convertido los estudios de redes. Estos, como naturales descendientes de la historiografía sobre familias y comunidades de mercaderes, apuntan innovadoras teorías y métodos de trabajo que insertan los estudios sobre los intercambios del modelo comercial y colonial hispanoamericano en la historia global. Si algo es necesario destacar de las redes de los siglos modernos es que mantienen su marco corporativo heredado de modelos tradicionales de comportamiento humano pero que ello no fue incompatible con la actuación en red en la nueva fase de las relaciones transnacionales que abrió la conexión de las flotas de Indias. Gracias a una valiosa narrativa histórica, sabemos que las redes de mercaderes de esta primera edad global dejaron un legado en aquellos lugares donde se asentaron, además de contribuir a la construcción de las infraestructuras portuarias y las instituciones. Sus pequeños negocios locales tenían una reverberación económica, social y cultural (y no menos política) a larga escala. La perspectiva metodológica de red apunta a recaer en el comportamiento de los agentes el análisis del devenir mercantil, pero ello no se puede deslindar de un análisis sobre su influencia en la articulación histórica de los territorios, enfocado dicho estudio desde la perspectiva de la espacialidad. Esta perspectiva se está introduciendo en las nuevas investigaciones comparadas de los imperios mercantiles, y de sus espacios socio-mentales compartidos.

Recibido el 7 de junio de 2018 Aceptado el 21 de agosto de 2018 


\section{Referencias bibliográficas}

Alcántara, Álvaro, «Un imperio también de agua. Puertos interiores, redes mercantiles y comercio de contrabando en las costas novohispanas, 1776-1795», Illes i Imperis 18, Barcelona, 2016, 77-106.

Alonso García, David, Mercados y mercaderes en los siglos XVI y XVII. Una historia global, Madrid, Síntesis, 2016.

Álvarez Nogal, Carlos, «Las remesas americanas en las finanzas de la Real Hacienda. La cuantificación del dinero de la Corona, 1621-1675», Revista de Historia Económica / Journal of Iberian and Latin American Economic History, 16 (2), 1998, 453-488.

Álvarez Nogal, Carlos, «Mercados o redes de mercaderes: el funcionamiento de la feria de Portobelo», en Böttcher, N. y Hausberger, B. (eds.), Redes y negocios globales en el mundo ibérico, siglos XVI-XVIII, México, El Colegio de México, Vervuert, 2011, 53-87.

Angulo Morales, Alberto y Aragón Ruano, Álvaro (eds.), Recuperando el Norte. Empresas, capitales y proyectos atlánticos en la economía imperial hispana, Bilbao, Universidad de País Vasco, 2016.

Aram, Bethany y Yun Casalilla, Bartolomé (eds.), Global Goods and the Spanish Empire, 1492-1824. Circulation, Resistance and Diversity, London, Palgrave Macmillan, 2014.

Aranda Pérez, Francisco José, Burgueses o ciudadanos en la España moderna, Universidad de Castilla La Mancha, 2003.

Bernal, Antonio Miguel, «La Casa de la Contratación de Indias. Del monopolio a la negociación mercantil privada (siglo XVI)», en Acosta Rodríguez, A.; González Rodríguez, A. y Vila Vilar, E. (eds.), La Casa de la Contratación y la navegación entre España y las Indias, Sevilla, CSIC y Universidad de Sevilla, 2003, 129-160.

Bernal, Antonio Miguel, España, proyecto inacabado: costes/beneficios del Imperio, Madrid, Marcial Pons, 2005.

Bernardo Ares, José Manuel de, «La nueva historia política: la interrelación estructural de biografías y procesos», en Bernardo Ares, J. M. de (coord.), La Sucesión de la Monarquía Hispánica, 1665-1725, Madrid, Sílex, Caja Sur, 2009, 11-22.

Bertrand, Michel; Guzzi-Heeb, Sandro y Lemercier, Claire, «Introducción: ¿En qué punto se encuentra el análisis de redes en Historia?», REDES. Revista hispana para el análisis de redes sociales, 21 (1), 2011, http://revista-redes. rediris.es/html-vol21/vol21_1.htm [Consultado: 08/02/2016].

Brendecke, Arndt, Imperio e información. Funciones del saber en el dominio colonial español, Madrid/Frankfurt, Iberoamericana/Vervuert, 2012.

Bustos Rodríguez, Manuel, Cádiz en el sistema atlántico. La ciudad, sus comerciantes y la actividad mercantil (1650-1830), Madrid, Sílex Ediciones, 2005. 
Bustos Rodríguez, Manuel, «Comercio y comerciantes en la Andalucía del Antiguo Régimen: estado de la cuestión y perspectivas», Obradoiro de Historia Moderna, 17, Santiago de Compostela, 2008, 43-76.

Bustos Rodríguez, Manuel, «Hacer de la necesidad virtud: el comercio textil de la Andalucía atlántica con América en el siglo XVIII», Estudis. Revista de Historia Moderna, 36, Valencia, 2010a, 87-130.

Bustos Rodríguez, Manuel, «La problemática acerca de los comerciantes de la Carrera de Indias», en Crespo Solana, A. (coord.), Comunidades transnacionales. Colonias de mercaderes extranjeros en el Mundo Atlántico, 1500-1830, Madrid, Doce Calles, 2010b, 29-45.

Bustos Rodríguez, Manuel, El Consulado de Cargadores a Indias en el siglo XVIII (1700-1830), Cádiz, Ediciones UCA, 2017.

Cachero Vinuesa, Montserrat, Deudos y parientes. Redes económicas y política matrimonial en la sociedad colonial, México, 1519-1544, [Almería], Editorial Círculo Rojo, 2013.

Caracausi, Andrea y Jegle, Christof (eds.), Commercial Networks and European Cities, 1400-1800, London/New York, Routledge, 2014.

Cardim, Pedro et al. (eds.), Polycentric Monarchies. How did Early Moderns Spain and Portugal Achieve and Maintain a Global Hegemony?, UK, Sussex Academic Press, Red Columnaria, 2012.

Castellano, Juan Luis y Dedieu, Jean-Pierre (dirs.), Réseaux, familles et pouvoirs dans le monde ibérique à la fin de l'Ancien Régime, Paris, CNRS Éditions, 1998.

Caula, Elsa, «Sociabilidades mercantiles y prácticas comerciales de los mercaderes vascos en el Buenos Aires virreinal», Caravelle, 101, 2013, 193-216.

Crailsheim, Eberhard, The Spanish connection. French and Flemish Merchant Networks in Seville (1570-1650), Wien, Böhlau, 2016.

Crespo Solana, Ana (coord.), Comunidades transnacionales. Colonias de mercaderes extranjeros en el Mundo Atlántico, 1500-1830, Madrid, Doce Calles, 2010a.

Crespo Solana, Ana, «Legal Strategies and Smuggling Mechanisms in the Trade with the Hispanic Caribbean by Foreign Merchants in Cadiz: The Dutch and Flemish Case, 1680-1750», Jahrbuch für Geschichte Lateinamerikas, 47, 2010b, 181-212.

Crespo Solana, Ana, «Geostrategy of a system? Merchant Societies and exchange Networks as connections centres in the Spanish Atlantic Trade in the First Global age», en Mukherjee, R. (ed.), Networks in the First Global age, 14001800, Delhi, Primus Book, 2011, 11-35.

Crespo Solana, Ana, «La Historia geográficamente integrada y los Sistemas de Información Geográfica (SIG): concepto y retos metodológicos», Tiempos Modernos, 26, 2013, 1-33, http://www.tiemposmodernos.org/tm3/index.php/ tm/article/view/331/373 [Consultado 14/08/2018]. 
Crespo Solana, Ana (ed.), Spatio-Temporal Narratives: Historical GIS and the Study of Global Trading Networks (1500-1800), London, Cambridge Scholars Publishing, 2014a.

Crespo Solana, Ana, «Diasporas and the Integration of "Merchant Colonies": Flemish and Dutch Networks in Early Modern Spain», Le Verger - bouquet V, 2014b, 1-21, http://cornucopia16.com/wp-content/uploads/2014/09/VBQV +-+CRESPO.pdf [Consultado: 01/05/2014].

Crespo Solana, Ana, «El más amplio Atlántico: redes mercantiles, comunidades globales», en Iglesias, J. J.; Pérez García, R. M.; Fernández Chaves, M. (eds.), Comercio y cultura en la Edad Moderna. Actas de las XIII reunión científica de la Fundación española de Historia Moderna, Sevilla, Editorial Universidad de Sevilla, 2015, 47-69.

Crespo Solana, Ana, «Merchants and the beating of a butterfly's wings: from local to global in the transfer of economic behavior models in the 18th century», en Herrero, M. y Kaps, K. (eds.), Merchants and Trade Networks in the Atlantic and the Mediterranean, 1550-1800. Connectors of commercial maritime systems, London/New York, Routledge, 2017, 83-106.

Curtin, Philip D., Cross-Cultural Trade in World History, Cambridge University Press, 1984.

Davis, Ralph, The Rise of the Atlantic Economy, Ithaca, Cornell University Press, 1973.

Domínguez-Domínguez, Citlalli, «Uniendo el comercio de la mar del norte y la mar del sur: La bioceanidad en el Caribe vista a través del eje Veracruz-Acapulco, en la segunda mitad del siglo XVI», en Padrón Reyes, L. y Domínguez, C. (coords.), El Caribe en el espacio atlántico, siglos XVI-XIX. Iberoamérica Social: Revista-red de estudios sociales, Número especial 2, Sevilla, 2018, 10-26.

Emmer, Pieter, «The Myth of Early Globalisation: The Atlantic Economy, 15001800», Nuevo Mundo Mundos Nuevos, 2008, http://journals.openedition.org/ nuevomundo/42173 [Consultado: 10/06/2017].

Fernández Chaves, Manuel F. y Gamero Rojas, Mercedes, «Flamencos en la Sevilla del siglo XVIII: las estrategias familiares, redes clientelares y comportamientos económicos», en Bravo Caro, J. J. y Sanz Sampelayo, L. (coords.), Población y grupos sociales en el Antiguo Régimen, Málaga, Universidad de Málaga, 2009, v. 1, 571-586.

Fujita, Masahisa; Krugman, Paul y Venables, Anthony J., The Spatial Economy: Cities, Regions and International Trade, Boston, The MIT Press, 1999.

Gamero Rojas, Mercedes, «Flamencos en la Sevilla del siglo XVII: actividades económicas entre Europa y América», en Iglesias Rodríguez, J. J. y García Bernal, J. J. (eds.), Andalucía en el mundo atlántico moderno. Agentes y escenarios, Madrid, Sílex, 2016, 287-310.

Games, Alison, «Atlantic History: Definitions, Challenges and Oportunities», The American Historical Review, 111 (3), 2006, 741-757. 
García Fernández, María Nélida, Comunidad extranjera y puerto privilegiado: Los británicos en Cádiz durante el siglo XVIII, Cádiz, Universidad de Cádiz, 2005.

García Fuentes, Lutgardo, El comercio español con América, 1650-1700, Sevilla, Diputación Provincial, 1982.

García Fuentes, Lutgardo, «Los vascos en la Carrera de Indias en la Edad Moderna: una minoría predominante», Temas americanistas, 16, Sevilla, 2003, 29-49.

García-Mauriño Mundi, Margarita, La pugna entre el Consulado de Cádiz y los jenizaros por las exportaciones a Indias (1720-1765), Sevilla, Universidad de Sevilla, 1999.

Gascón, Margarita, «Comerciantes y redes mercantiles del siglo XVII en la frontera sur del Virreinato del Perú», Anuario de Estudios Americanos, 57-2, Sevilla, 2000, 413-448.

Gervais, Pierre; Lemarchand, Yannick y Margairaz, Dominique (eds.), Merchants and Profit in the Age of Commerce, 1680-1830, London, Pickering \& Chatto, 2014.

Granovetter, Mark, «The Strenght of Weak Ties: A Network Theory Revisited», Sociological Theory, 1, 1983, 201-233.

Gruzinski, Serge, Les quatre parties du monde. Histoire d'une mondialisation, Paris, La Martinière, 2004.

Guerrero Elecalde, Rafael, Las élites vascas y navarras en el gobierno de la monarquía borbónica. Redes sociales, carreras y hegemonía en el siglo XVIII (1700-1746), Bilbao, Universidad del País Vasco, 2012.

Guimerá Ravina, Agustín, «La burguesía mercantil canaria en la etapa del libre comercio (1765-1824)», en Bernal, A. M. (coord.), El comercio libre entre España y América Latina (1765-1824), Madrid, Fundación Banco Exterior, 1987, 261-288.

Haggerty, Sheryllynne, «Structural holes and bad ideas: Liverpool's Atlantic trade networks in the early-eighteenth century», en Herrero, M. y Kaps, K. (eds.), Merchants and Trade Networks in the Atlantic and the Mediterranean, 15501800, London/New York, Routledge, 2017, 237-255.

Herrero Sánchez, Manuel et al. (coords.), Génova y la Monarquía Hispánica (1528-1713), Génova, Società Ligure di Storia Patria, 2011.

Herrero Sánchez, Manuel y Kaps, Klemens (eds.), Merchants and Trade Networks in the Atlantic and the Mediterránean, 1550-1800. Connectors of commercial maritime systems, London/New York, Routledge, 2017.

Ibarra, Antonio y Valle Pavón, Guillermina del (coords.), Redes sociales e instituciones comerciales en el imperio español, siglos XVII a XIX, México, Instituto Mora, 2007.

Iglesias Rodríguez, Juan José y García Bernal, José Jaime (eds.), Andalucía en el mundo atlántico moderno. Agentes y escenarios, Madrid, Sílex, 2016. 
Iglesias Rodríguez, Juan José; García Bernal, José J. y Díaz Blanco, José Manuel (eds.), Andalucía en el mundo atlántico moderno. Ciudades y redes, Madrid, Sílex, 2018.

Imízcoz Beunza, Jose María, «Familia y redes sociales en la Edad Moderna», en Lorenzo Pinar, F. J. (ed.), La familia en la Historia, Salamanca, Universidad de Salamanca, 2009, 135-186.

Kaps, Klemens y Komlosy, Andrea, «Centers and Peripheries Revisited: Polycentric Connections or Entangled Hierarchies?», Review (Fernand Braudel Center), 36 (3-4), Binghamton, 2013, 237-264.

Kaps, Klemens, «Small but powerful: networking strategies and the trade business of Habsburg-Italian merchants in Cadiz in the second half of the eighteenth century», Journal European Review of History: Revue Européenne d'Histoire, 23 (3), 2016, 427-455.

Lamikiz, Xabier, Trade and Trust in the Eighteenth-Century Atlantic World. Spanish Merchants and their Overseas Networks, UK, The Boydell Press, 2010.

Latasa, Pilar, «Negociar en red: familia, amistad y paisanaje. El virrey Superunda y sus agentes en Lima y Cádiz (1745-1761)», Anuario de Estudios Americanos, 60-2, Sevilla, 2003, 463-492.

Marcelín-Jiménez, Ricardo, «Luciérnagas e Internet. La magia de las redes complejas», en Laguna Sánchez, G. et al. (coords.), Complejidad y Sistemas complejos. Un acercamiento multidimensional, México, Editora C3, 2016, 99-110.

Mauro, Frederic, «Merchant communities, 1350-1750», en Tracy, J. D. (ed.), The Rise of Merchant Empires. Long-distance trade in the Early Modern world, 1350-1750, Cambridge University Press, 1990, 255-286.

Mira Caballos, Esteban, La Armada Guardacostas de Andalucía y la defensa de la Carrera de Indias (1521-1550), Sevilla, Muñoz Moya Editor, 1998.

Molina Puche, Sebastián e Irigoyen López, Antonio (eds.), Territorios distantes, comportamientos similares. Familias, redes y reproducción social en la Monarquía Hispánica (siglos XIV-XIX), Murcia, Editum, 2009.

Oliva Melgar, José María, El monopolio de Indias en el siglo XVII y la economía andaluza. La oportunidad que nunca existió, Huelva, Universidad de Huelva, 2004.

Oostindie, Gert y Roitman, Jessica V. (eds.), Dutch Atlantic Connections, 16801800: Linking Empires, Bridging Borders, Leiden, Boston, Brill, 2014.

Otte, Enrique, «Empresarios españoles y genoveses en los comienzos del comercio trasatlántico: la avería de 1507», Revista de Indias, 93-94, Madrid, 1963, 519-530.

Parry, J. H., The Spanish Seaborne Empire, Berkeley, University of California Press, 1990.

Pérez Sarrión, Guillermo, La península comercial. Mercado, redes sociales y Estado en España en el siglo XVIII, Madrid, Marcial Pons, 2012. 
Picazo Muntaner, Antoni, «Redes invisibles: cooperación y fraude en el comercio Manila-Acapulco», Anales del Museo de América, 19, Madrid, 2011, 140-152.

Pieper, Renate y Schmidt, Peer (eds.), Latin America and the Atlantic World. El mundo atlántico y América Latina. Essays in honor of Horst Pietschmann, Köln, Böhlau, 2005.

Polónia, Amélia y Rivera Medina, Ana M. (eds.), La gobernanza de los puertos atlánticos, siglos XIV-XX. Políticas y estructuras portuarias, Madrid, Casa de Velázquez, 2016.

Recio Morales, Óscar (ed.), Redes de nación y espacios de poder. La comunidad irlandesa en España y la América española, 1600-1825, Valencia, Albatros, Ministerio de Defensa, 2012.

Ruiz Rivera, Julián B., El Consulado de Cádiz: matrícula de comerciantes, 17301823, Cádiz, Diputación Provincial, 1988.

Ruiz Rivera, Julián B., «La colonia mercantil catalana en Cádiz», Temas americanistas, 8, Sevilla, 1990, 15-24.

Ruiz Rivera, Julián B., «Presencia navarra en el Cádiz del monopolio», Príncipe de Viana. Anejo, 15, Pamplona, 1993, 49-75.

Sanz Ayán, Carmen, Un banquero en el Siglo de Oro: Octavio Centurión, el financiero de los Austrias, Madrid, La Esfera de los Libros, 2015.

Schulte Beerbühl, Margrit, «Merchant Empires: Mercaderes hamburgueses en Londres y sus redes de comercio internacional (1660-1815)», en Crespo Solana, A. (coord.), Comunidades transnacionales. Colonias de mercaderes extranjeros en el Mundo Atlántico, 1500-1830, Madrid, Doce Calles, 2010, 103-121.

Socolow, Susan, «La burguesía comerciante de Buenos Aires en el siglo XVIII», Desarrollo Económico, 70, Buenos Aires, 1978, 205-216.

Stein, Stanley J. y Stein, Barbara H., Plata, comercio y guerra. España y América en la formación de la Europa moderna, Barcelona, Crítica, 2002.

Studnicki-Gizbert, Daviken, «From Agents to Consulado: Commercial Networks in Colonial Mexico, 1520-1590 and Beyond», Anuario de Estudios Americanos, 57-1, Sevilla, 2000, 41-68.

Subrahmanyam, Sanjay (ed.), Merchant Networks in the Early Modern World, 1450-1800. Vol. 8. An Expanding World: The European Impact on World History, 1450 to 1800, UK, Routledge, 1996.

Trivellato, Francesca, The Familiarity of Strangers. The Sephardic Diaspora, Livorno, and Cross-Cultural Trade in the Early Modern Period, Yale University Press, 2009.

Valle Pavón, Guillermina del, Finanzas piadosas y redes de negocios. Los mercaderes de la ciudad de México ante la crisis de Nueva España, 1804-1808, México, Instituto Mora, 2012. 
Van Young, Eric, «Social Networks: A final Comment», en Böttcher, N.; Hausberger, B. e Ibarra, A. (coords.), Redes y negocios globales en el mundo ibérico, siglos XVI-XVIII, México, El Colegio de México, 2011, 289-311.

Vila Vilar, Enriqueta, Los Corzo y los Mañara: tipos y arquetipos del mercader con América, Sevilla, Escuela de Estudios Hispano-Americanos, CSIC, 1991.

Vila Vilar, Enriqueta y Kuethe, Allan J. (eds.), Relaciones de poder y comercio colonial, Sevilla/Texas, CSIC/Texas-Tech University, 1999.

Vila Vilar, Enriqueta, Hispanismo e Hispanización: El Atlántico como nuevo Mare Nostrum, Madrid, Real Academia de la Historia, 2012a.

Vila Vilar, Enriqueta, «Redes mercantiles y sociales entre Sevilla y Lima», en Vila Vilar, E. y Lacueva Muñoz, J. (coords.), Mirando las dos orillas: intercambios mercantiles, sociales y culturales entre Andalucía y América, Sevilla, Fundación de Buenas Letras, 2012b, 183-207.

Villar García, María Begoña y Pezzi Cristóbal, Pilar (eds.), Los extranjeros en la España Moderna. Actas del I Coloquio Internacional, Málaga, Universidad de Málaga, 2003, 2 tomos.

Wachowicz, Monica y Owens, J. B., «Dynamics of Trade Networks: The Main Research Issues on Space-Time Representations», en Crespo Solana, A. y Alonso García, D. (coords.), Self-Organizing Networks and GIS Tools. Cases of Use for the Study of Trading Cooperation (1400-1800), Special Issue of Journal of Knowledge Management, Economics and Information Technology, June 2012, 53-80.

Yun Casalilla, Bartolomé (dir.), Las Redes del imperio. Élites sociales en la articulación de la Monarquía Hispánica, 1492-1714, Madrid, Marcial Pons Historia, Universidad Pablo de Olavide, 2009.

Yuste López, Carmen, Comerciantes mexicanos en el siglo XVIII, México, UNAM, 1991.

Yuste López, Carmen, Emporios transpacíficos. Comerciantes mexicanos en Manila 1710-1815, México, UNAM, 2007. 